\title{
Measurements of Aging Effects of Facial Color Distribution and Applications
}

\author{
J. Shibatani, M. Masano*, T. Ishida*, \\ M. Shinoda*, S. Yuasa*
}

The purpose of this study was to enable to characterize the facial color change due to aging, and to substantiate the effectiveness of color control by makeups.

We measured skin color of 5 facial areas of 300 Japanese women between 16 to 68 years old, during April. The aging effects of facial color were characterized by the color distribution in different facial areas. It was found that the distribution came narrower with aging, and the facial color became more uniform and darker. The most natable color change due to aging occured in cheek area of women between twenties and thirties, and the least color change was observed in neck area.

To study the coloring effect of base makeup, we also measured the skin colors of 25 Women before and after applying several different cosmetics, and found that the color of skin after the application could be predicted from a proper function of bare skin color by a regression analysis.

By combining the information from the study on the apparent change in color distribution due to aging with the information on skin color change made possible through the application of makeups, we were able to develop means by which "youthful appearance" could be optimized.

Using the computer graphics, we were able to demonstrate to a customer the change in facial appearance prior to actual application of makeups.

\section{Introduction}

The desire to look young and to have a beautiful complexion is universal. However, aging inevitably causes various internal and external changes in human body. Externally, aging causes changes in skin activity, skin surface shape and skin color. Physiologically, aging prolongs the turnover time of epiderma cell, and reduces sebaceous secretion. The most obvious change on skin surface is the appearance of wrinkles. In addition, facial colors become darker, and stains may appear.

Makeup cosmetics are used to modify and control the color, luster and texture of skin to make it more attractive and appear youthful. Although skilled makeup artists know how to render youthful

R \& D Laboratories POLA Corporation; 648 Yayoi-cho, Shizuoka

* Beauty Research Department POLA Corporation; 2-3-2 Nishigotanda, Shinagawa-ku, Tokyo look through makeup application, they solely depend on their experience and intuition to achieve the desired appearance. Naturally, it would be desirable to provide less skilled customers a reliable guide to select and use makeups to acquire youthful look. From a scientific view point, this will require information on characterization of skin aging effects as well as the effects of makeups on skin color.

However, accurate and objective measurements of aging effects on skin are very difficult, and there are only a few published reports on this subject. Previously, we reported a study on measurements of aging effects of skin elasticity at the 12th Congress of IFSCC in Paris.

In this study, we focussed our work on the aging effects of women's facial color distribution as we have found no published work in this area.

After developing an instrumental method, we conducted an extensive measurements of skin color on different areas of a large number of women to 
characterize chromatic changes due to aging.

In order to desidn a program for computer graphics which could be used to predict changes in skin color after makeup application, we also made a complete study on the coloring effects of makeup preparations on human skin.

\section{Experiments}

\section{Instrument}

For this study it was desirable to develop a technique which allows precise and quick measurements of skin color. Fig. -1 is a diagram of measuring device used in this research. It consists of a tungsten lamp, optical filters and silicon photocells. This is similar to our original instrument, but was modified to make it smaller and more precice.

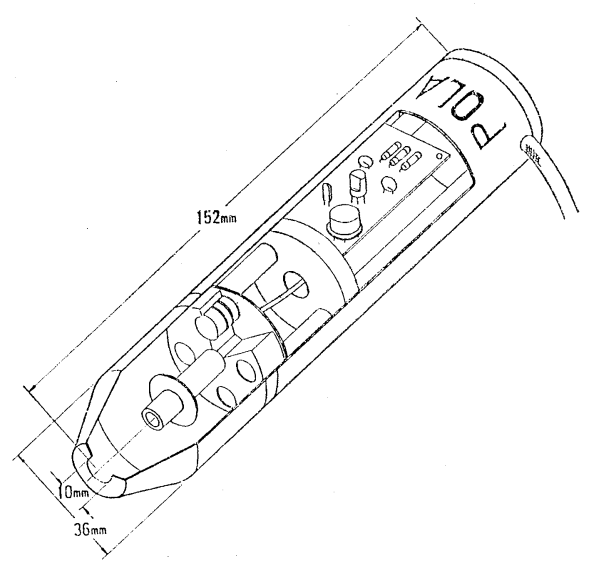

Fig. 1 Optical diagram of measuring device

The accuracy of measurements was assured with the spectrophotometer by compaing the data of measurements of various skin colors and standard color cards. The color data $\mathrm{X}, \mathrm{Y}$ and $\mathrm{Z}$ were converted to Munsell Renotation System, Hue, Value and Chroma.

\section{Subjects}

16 to 68 years old, Japanese women (The age distribution of the subjects is shown in Fig.-2).

\section{Measured areas}

Fig. -3 shows the five measured areas, forehead, blushing area of cheek, center of cheek, side of face

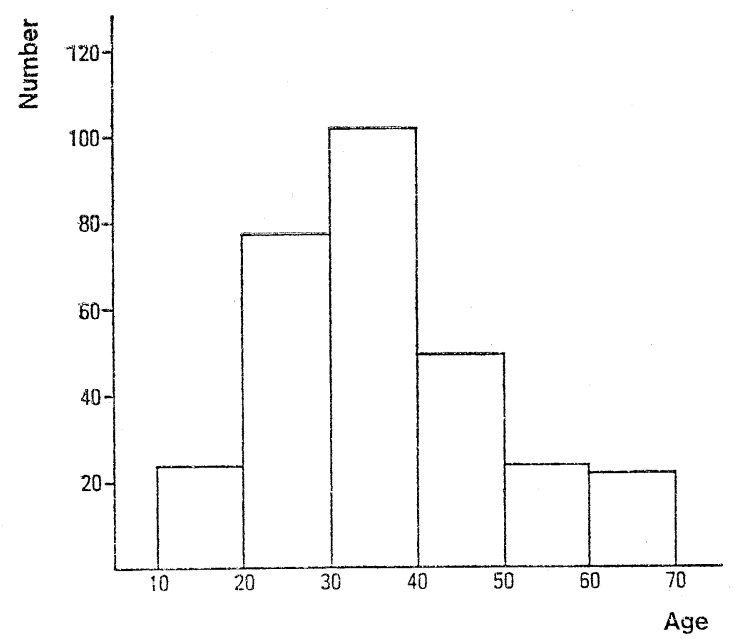

Fig.2 Distribution of subjects

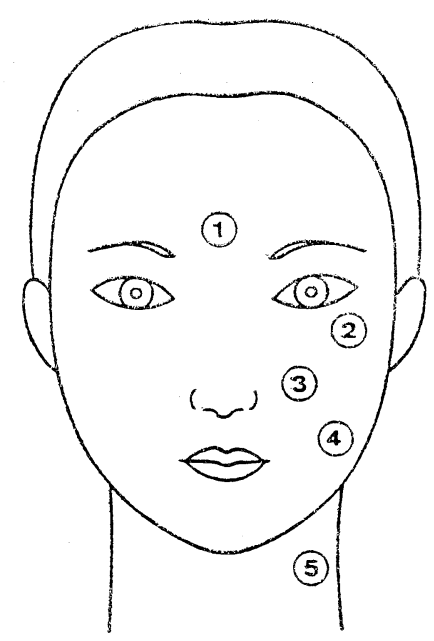

Fig.3 Measured area

and neck. These areas, one through five, were chosen from our previous study.

4. Measuring date

April 18 to 24, 1983

\section{Measuring condition}

At 10 to 20 minutes after cleansing, 3 time per a area, at room temperature.

\section{Results and Discussion}

The aging effects of facial colors were most 

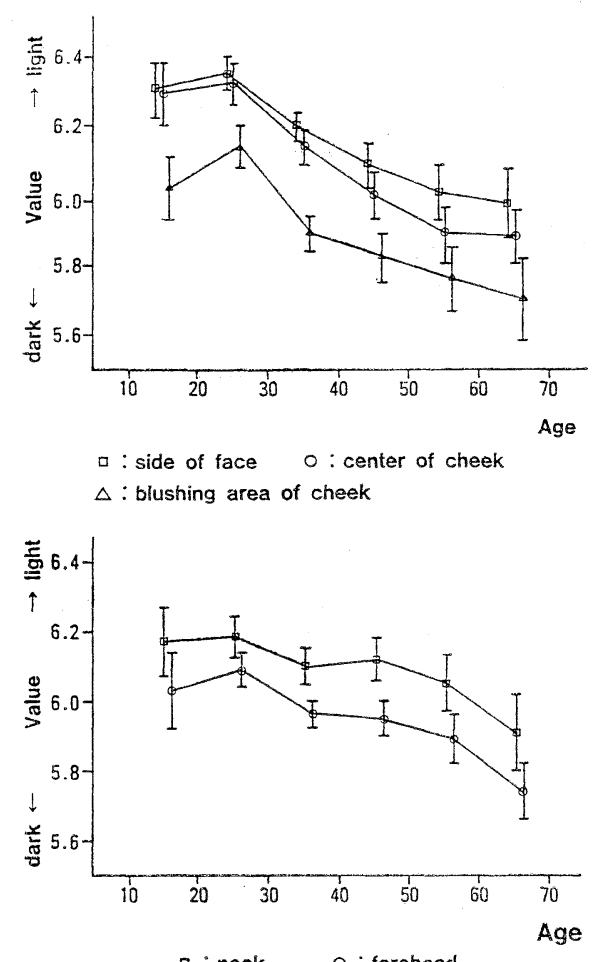

Fig.4 Facial color change with aging

apparent with respect to Munsell Value, which represents lightness. The change of lightness of five areas with aging is shown in Fig.-4. Lightness deceases with aging, at each areas. The most notable colar change due to aging occures in cheek area. The change between twenties and thirties is the greatest. From thirties on, facial color changes gradually.

The lightest areas of the face are the side of face and the center of cheek. The forehead is darkest.

The change of Hue with aging is shown in Fig. -5 . Hue shows almost no change. Blushing area of cheek is naturally the reddest, and neck area is the yellowest.

The change of Chroma due to aging is shown in Fig. -6. The Chroma increases with aging, especially in cheek area, but increases slightly in forehead and neck area. The most saturated area is the blushing area of cheek.

Thus, facial colors tend to become darker and
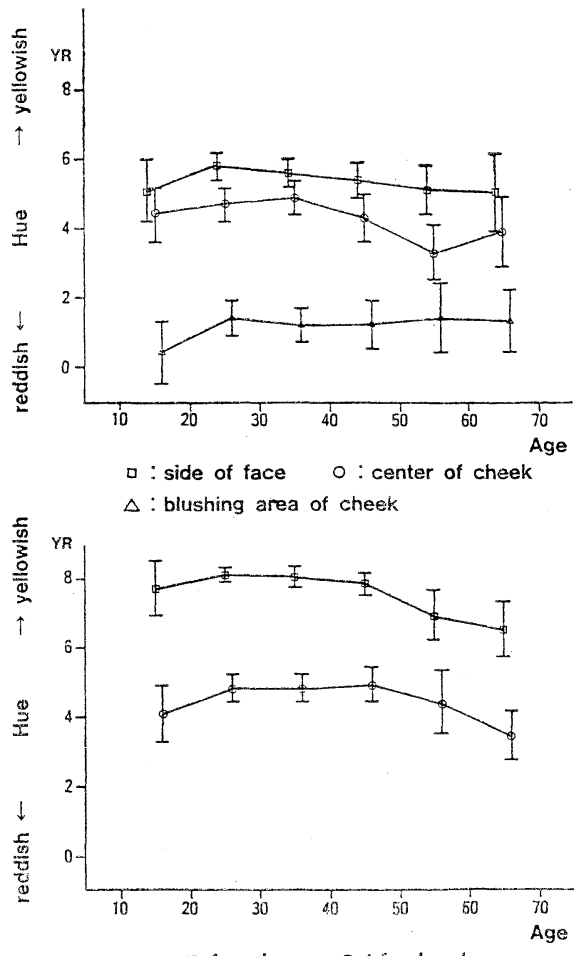

Fig.5 Facial color change with aging

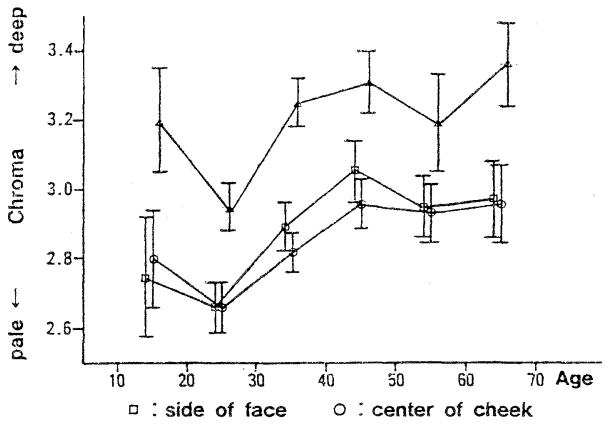

$\Delta$ : blushing area of cheek

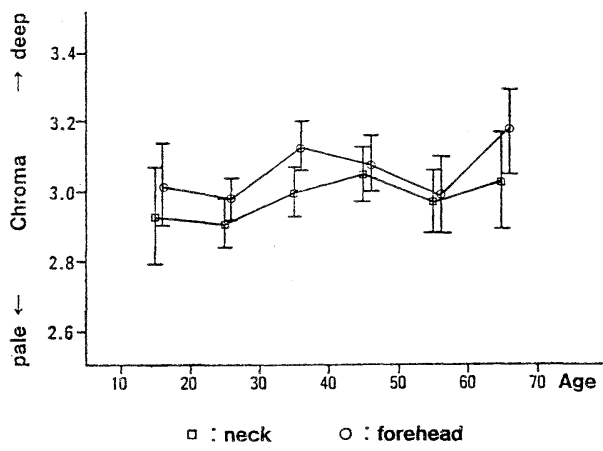

Fig. 6 Facial color change with aging 

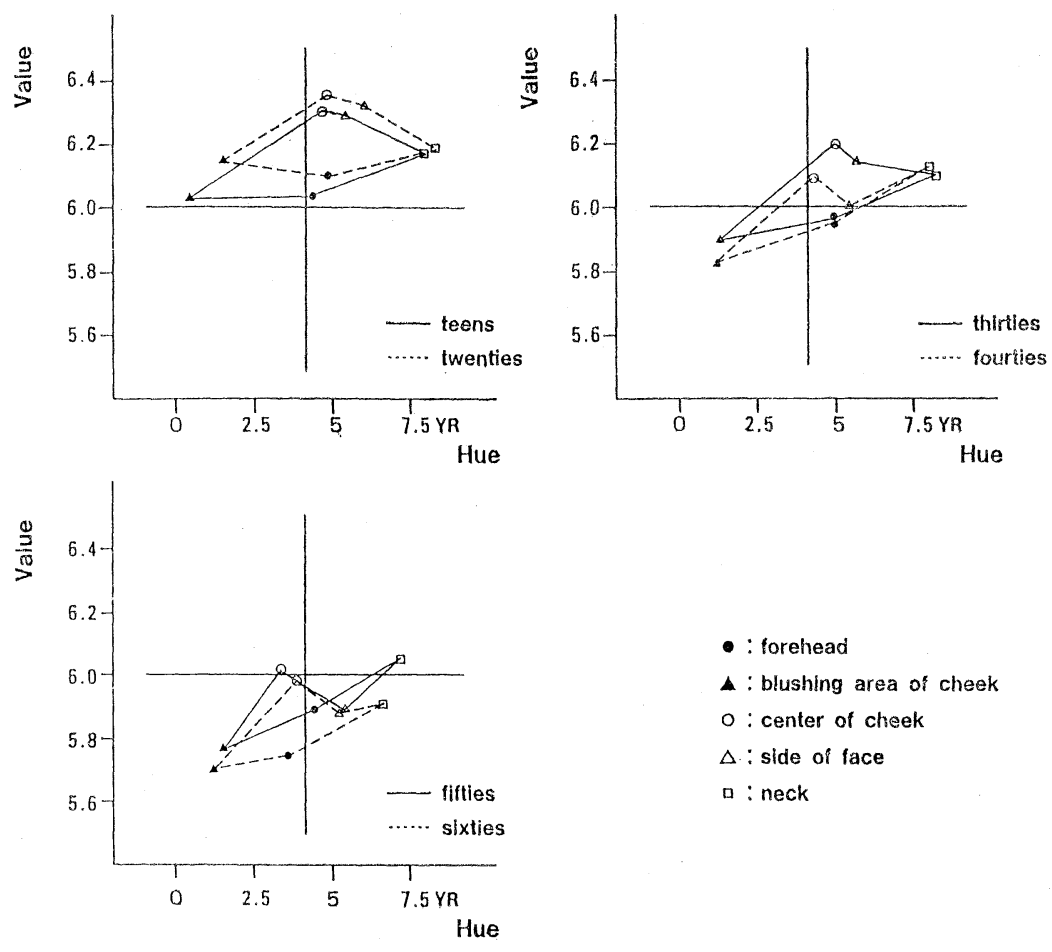

Fig.7 Facial color distribution

deeper with aging.

Fig. -7 shows the facial color distribution. The colors of five facial areas at different ages were plotted on the color map of Hue and Value. The facial color distribution, that is, color difference between the different facial areas is larger in younger subjects, but the difference becomes smaller and colors of each areas becomes more uniform with aging.

Facial colors become darker with aging, but neck area is the least affected area. As the result, the neck area of older person is fairest area of the head. The color of neck area is relatively unaffected by the change in blood circulation and suntan compared to other facial areas.

To study the coloring effect of makeups, we also measured the skin colors of 25 women before and after applying several different cosmetics, and found that the color of skin after application could be predicted from a proper function of bare skin color by regression analysis. Fig. -8 is an example. It shows the facial colors predicted by applying a dark ocher and light pink foundation creams. The change

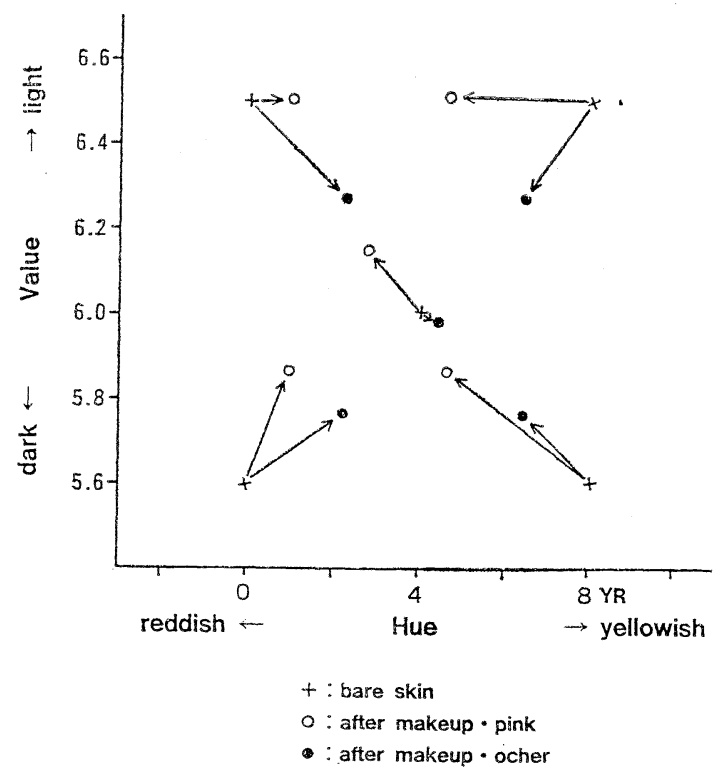

Fig. 8 Colors of bare skin and predicted colors after makeup 


\section{粧 技 衿 第19巻第 1 号 IFSCC 特集号}

of color is shown with arrows originating from bare skin colors.

By using the information from the study on apparent change in color distribution due to aging with the information on skin color changes through the application of makeups, we were able to develop means by which youthful appearance could be optimized. With the originally developed computer graphics system, we simulated the effects of color control by makeups. This system consists of 8 bit microprocessor, a color monitor, a light pen and a tablet digitizer. The color monitor has resolution as high as $256 \times 256$ pixels and about $16,700,000$ colors.

We also used the subsystem to input picture data into the main computer. This consists of a videocamera, a color graphic terminal and a micro computer.

The system calculates color changes required to render a youthful look, and selectes the color of makeups. Then it displays simulated faces on a color monitor. In this way we were able to demonstrate to a custumer the coloring effects of makeups without actual application of makeups.

\section{Reference}

1) M.Okada, T. Ishida, S. Yuasa, Measurements of Skin Elasticity and Slip; Application in Efficacy Substatiation. J. Soc. Cosmet. Chem. 17, 7-13 (1983)

2) J. Shibatani, M. Masano, S. Kuroda, N. Nakajima, S.Yuasa, Studies on Skin Color and Makeup Effects (1) Development of Skin Color Measuring Method and Application in Efficacy Substantiation. J. Soc. Cosmet. Chem. 17, 138-145 (1983)

3) T.Ishida, M.Shinoda, J. Shibatani, Y. Yuasa Development of Makeup Simulator by Appling Computer Graphics. J. Soc. Cosmet. Chem. 18. 91-95 (1984) 